\title{
Radial breathing vibration of double-walled carbon nanotubes subjected to
}

\author{
pressure
}

\section{Xiao-Wen Lei}

Interdisciplinary Graduate School of Science \& Technology, Shinshu University, 3-15-1 Tokida, Ueda, Nagano 386-8567, Japan

Toshiaki Natsuki

Department of Functional Machinery \& Mechanics, Shinshu University,

3-15-1 Tokida, Ueda, Nagano 386-8567, Japan

Jin-Xing Shi

Interdisciplinary Graduate School of Science \& Technology, Shinshu University, 3-15-1 Tokida, Ueda, Nagano 386-8567, Japan

Qing-Qing $\mathrm{Ni}^{*}$

Department of Functional Machinery \& Mechanics, Shinshu University, 3-15-1 Tokida, Ueda, Nagano 386-8567, Japan

*Corresponding author

E-mail: niqq@shinshu-u.ac.jp; Tel: +81-0268-21-5438; Fax: +81-0268-21-5438 


\begin{abstract}
A theoretical vibrational analysis of the radial breathing mode (RBM) of double-walled carbon nanotubes (DWCNTs) subjected to pressure is presented based on an elastic continuum model. The results agree with reported experimental results obtained under different conditions. Frequencies of the RBM in DWCNTs subjected to increasing pressure depend strongly on circumferential wave numbers, but weakly on the aspect ratio and axial half-wave numbers. For the inner and outer tubes of DWCNTs, the frequency of the RBM increases obviously as the pressure increases under different conditions. The range of variation is smaller for the inner tube than the outer tube.
\end{abstract}

\title{
Key words:
}

radial breathing mode; carbon nanotube; vibration; pressure 


\section{Introduction}

The study of vibration in carbon nanotubes (CNTs) is currently a major topic of interest [1-6] that increases understanding of their dynamic mechanical behavior. Raman spectroscopy has been widely recognized as a powerful nondestructive technique for characterizing the structural properties of CNTs because of better sensitivity and lower cost than other methods [7]. Raman spectra contain different features including the radial breathing mode (RBM), where all of the carbon atoms are subject to an in-phase radial displacement, the G-band, where neighboring atoms move in opposite directions along the surface of the tube as in $2 \mathrm{D}$ graphite, the dispersive disorder-induced D-band and its second order related harmonic G'-band. Of these, the RBM appears at the lowest frequency but is the strongest feature observed. In the RBM, all of the carbon atoms in a CNT move in a radial direction synchronously as if the tube is "breathing" $[7,8]$. This mode is unique to CNTs, and is not observed in other carbon systems [9].

Application of pressure to condensed matter systems is an ideal way to continuously modify the bonding properties of a solid, which affect virtually all of the properties of a material [10]. In the context of CNTs, pressure studies are motivated by the needs to investigate the mechanical stability, pressure-induced phase transitions like vibrational 
characteristics, and the effects of intertube interactions. The vibrational properties of nanotubes in a high-pressure diamond anvil cell (DAC) can be readily investigated by Raman spectroscopy [11].

The frequency of the RBM of CNTs under pressure has mainly been probed using Raman spectroscopy [12-15]. Schlecht et al. [12] studied isolated single-walled carbon nanotubes (SWCNTs) under pressure experimentally and found that the frequency dependence of the optical mode in bundled and isolated SWCNTs was identical. Using generalized tight-binding molecular dynamics, Venkateswaran et al. [13, 14] showed that van der Waals (vdW) interactions make an important contribution to the frequency of the RBM of bundled and unbundled CNTs and impact its pressure dependence strongly. During consideration of the structure characteristics of CNTs, Lebedkin et al. [15] demonstrated that it is possible to study pressure effects on individual unbundled SWCNTs using a DAC and a sensitive Raman spectrometer. The position of the RBM has been reported to be sensitive to excitation wavelength $[16,17]$, external pressure $[18$, 19], dopant type [20], and the size of the nanotube bundle [21]. Arvanitidis et al. [11] used detailed pressure Raman experiments to determine the strength of intra-tube interactions of bundled double-walled carbon nanotubes (DWCNTs). Gadagkar et al. [22] focused on the responses of the inner and outer tubes of DWCNTs, and 
investigated the behavior of DWCNT bundles under pressure. Puech et al. $[23,24]$ examined the stability of DWCNTs under hydrostatic pressures, and compared Raman spectra obtained under different hydrostatic pressures using two different pressure media.

Computational simulation has been regarded as a powerful tool for predicting the frequency of the RBM of CNTs under hydrostatic pressure, comparing with the difficulty of the experiment. Lawler and Kurti et al. $[25,26]$ presented first-principle calculations for the RBM of bundled SWCNTs. A study exploring the frequencies and mode shapes of the RBM of various CNTs using a modified molecular structural mechanics model was reported by Cheng et al. [9]. Based on a multiple-elastic shell model, Wang et al. [27] showed that the frequencies of the RBM of MWCNTs generally increase as the external pressure increases, and the effects of pressure are associated with RBMs of different frequency for MWCNTs with innermost tubes of different diameter. Recent reviews focusing on Raman spectroscopy on CNTs [7] and CNTs under pressure [8] summarized past achievements in the field and highlighted promising directions for future development, especially in experimental studies.

In this letter, the frequency of the RBM of CNTs subjected to pressure, is studied using an elastic continuum mechanics model. DWCNTs can be considered a kind of 
MWCNT where the interlayer interaction between the inner and outer nanotubes is generally turbostratic $[7,8]$. The influences of the axial half-wave number $m$, circumference wave number $n$, nanotube radius, aspect ratio $\mathrm{L} / \mathrm{D}$ of nanotubes on the frequency of the RBM of the inner and outer tubes in DWCNTs under varying pressure are considered. Compared to previous results obtained from experimental investigations, the continuum shell model can be used to predict the frequency of the RBM of CNTs under pressure reasonably. This investigation will be helpful in nanodevice technologies such as nanoprobes and nanosensors.

2. Theoretical approach

\subsection{Governing equations of DWCNTs under pressure}

A continuum elastic shell model (Fig. 1) was used to analyze the characteristics of the $\mathrm{RBM}$ of CNTs subjected to pressure. The cylindrical shell is designated as a coordinate system $(x, \theta, z)$. The coordinates $x, \theta$, and $z$ refer to the axial, circumferential and radial directions, respectively. The displacements of CNTs are $u, v$ and $w$ corresponding to the $x, \theta$, and $z$ directions, respectively. The dimensions of the nanotubes are defined as the thickness $h$, radius $R$, length $L$ and Poisson's ratio $v$.

Based on our previous work [28, 29], the external forces $N$ and moment $M$ resultants 
are respectively defined as

$$
\begin{aligned}
& \left\{N_{x}, N_{\theta}, N_{x \theta}, N_{\theta x}\right\}=\frac{E h}{1-v^{2}}\left\{\epsilon_{1}+v \epsilon_{2}, \epsilon_{2}+v \epsilon_{1}, \gamma(1-v) / 2, \gamma(1-v) / 2\right\} \\
& \left\{M_{x}, M_{\theta}, M_{x \theta}, M_{\theta x}\right\}=-D\left\{\chi_{x}-v \chi_{\theta}, \chi_{\theta}+v \chi_{x},(v-1) \chi_{x \theta},(1-v) \chi_{x \theta}\right\}
\end{aligned}
$$

where $E$ is Young' modulus of CNTs.

$$
\begin{aligned}
D & =\frac{E h^{3}}{12\left(1-v^{2}\right)} & \\
\epsilon_{1}=\frac{\partial u}{\partial x} & \epsilon_{2}=\frac{\partial v}{R \partial \theta}-\frac{w}{R} & \gamma=\frac{\partial u}{R \partial \theta}+\frac{\partial v}{\partial x} \\
\chi_{x}=\frac{\partial^{2} w}{\partial x^{2}} & \chi_{\theta}=\frac{1}{R^{2}}\left(\frac{\partial v}{\partial \theta}+\frac{\partial^{2} w}{\partial \theta^{2}}\right) & \chi_{x \theta}=\frac{1}{R}\left(\frac{\partial v}{\partial x}+\frac{\partial^{2} w}{\partial x \partial \theta}\right)
\end{aligned}
$$

We considered the vibration of CNTs in the radial direction according to Love's first approximation shell theory. Therefore, the equilibrium equations of the three external forces and moments acting on CNTs are given by

$$
\begin{aligned}
& R \frac{\partial N_{x}}{\partial x}+\frac{\partial N_{\theta x}}{\partial \theta}-N_{\theta}\left(\frac{\partial^{2} v}{\partial x \partial \theta}-\frac{\partial w}{\partial x}\right)=0 \\
& \frac{\partial N_{\theta}}{\partial \theta}+R \frac{\partial N_{x \theta}}{\partial x}-\frac{\partial M_{\theta}}{R \partial \theta}+\frac{\partial M_{x \theta}}{\partial x}=0 \\
& \frac{\partial^{2} M_{\theta x}}{\partial x \partial \theta}+R \frac{\partial^{2} M_{x}}{\partial x^{2}}+\frac{\partial^{2} M_{\theta}}{R \partial \theta^{2}}-\frac{\partial^{2} M_{x \theta}}{\partial x \partial \theta}+N_{\theta}\left(1+\frac{\partial v}{R \partial \theta}+\frac{\partial^{2} w}{R \partial \theta^{2}}\right)+p_{h} R \\
& =R \rho h \frac{\partial^{2} w}{\partial t^{2}}
\end{aligned}
$$

where $p_{h}$ is the external pressure acting on the tube, which is shown in Fig. 2(a). $\rho$ is the density of CNTs. According to theory [30], by substituting Eqs. (1) - (5) into Eqs. (6) (8), we obtain

$$
\frac{\partial^{2} u}{\partial x^{2}}+\frac{(1-v)}{2 R^{2}} \frac{\partial^{2} u}{\partial \theta^{2}}+\frac{(1+v)}{2 R} \frac{\partial^{2} v}{\partial x \partial \theta}+p_{h} \Phi \frac{\partial^{2} v}{\partial x \partial \theta}-\frac{v}{R} \frac{\partial w}{\partial x}-p_{h} \Phi \frac{\partial w}{\partial x}=0
$$




$$
\begin{gathered}
\frac{(1+v)}{2 R} \frac{\partial^{2} u}{\partial x \partial \theta}+\frac{1}{R^{2}} \frac{\partial^{2} v}{\partial \theta^{2}}+\frac{(1-v)}{2} \frac{\partial^{2} v}{\partial x^{2}}+\beta \frac{1}{R^{2}} \frac{\partial^{2} v}{\partial \theta^{2}}+(1-v) \beta \frac{\partial^{2} v}{\partial x^{2}}-\frac{1}{R^{2}} \frac{\partial w}{\partial \theta} \\
+\beta \frac{\partial^{3} w}{\partial x^{2} \partial \theta}+\frac{\beta}{R^{2}} \frac{\partial^{3} w}{\partial \theta^{3}}=0 \\
\frac{v}{R} \frac{\partial u}{\partial x}+\frac{1}{R^{2}} \frac{\partial v}{\partial \theta}-(2-v) \beta \frac{\partial^{3} v}{\partial x^{2} \partial \theta}-\frac{\beta}{R^{2}} \frac{\partial^{3} v}{\partial \theta^{3}}-\frac{1}{R^{2}} w-2 \beta \frac{\partial^{4} w}{\partial x^{2} \partial \theta^{2}}-R^{2} \beta \frac{\partial^{4} w}{\partial x^{4}} \\
-\frac{\beta}{R^{2}} \frac{\partial^{4} w}{\partial \theta^{4}}-\frac{p_{h} \Phi}{R} w-\frac{p_{h} \Phi}{R} \frac{\partial^{2} w}{\partial \theta^{2}}-\rho h \Phi \frac{\partial^{2} w}{\partial t^{2}}=0
\end{gathered}
$$

where $\Phi=\left(1-v^{2}\right) / E h$ and $\beta=h^{2} / 12 R^{2}$. To simplify the calculation, Eqs. (9) $-(11)$ can be

rewritten as

$$
\left[\begin{array}{lll}
L_{11} & L_{12} & L_{13} \\
L_{21} & L_{22} & L_{23} \\
L_{31} & L_{32} & L_{33}
\end{array}\right]\left\{\begin{array}{c}
u \\
v \\
w
\end{array}\right\}=0
$$

where $L_{i j}$ are the differential operators given as

$$
\begin{aligned}
& L_{11}=\frac{\partial^{2}}{\partial x^{2}}+\frac{(1-v)}{2 R^{2}} \frac{\partial^{2}}{\partial \theta^{2}} \\
& L_{12}=\frac{(1+v)}{2 R} \frac{\partial^{2}}{\partial x \partial \theta}+p_{h} \Phi \frac{\partial^{2}}{\partial x \partial \theta} \\
& L_{13}=-\frac{v}{R} \frac{\partial}{\partial x}-p_{h} \Phi \frac{\partial}{\partial x} \\
& L_{21}=\frac{(1+v)}{2 R} \frac{\partial^{2}}{\partial x \partial \theta} \\
& L_{22}=\frac{1}{R^{2}} \frac{\partial^{2}}{\partial \theta^{2}}+\frac{(1-v)}{2} \frac{\partial^{2}}{\partial x^{2}}+\beta \frac{1}{R^{2}} \frac{\partial^{2}}{\partial \theta^{2}}+(1-v) \beta \frac{\partial^{2}}{\partial x^{2}} \\
& L_{23}=-\frac{1}{R^{2}} \frac{\partial}{\partial \theta}+\beta \frac{\partial^{3}}{\partial x^{2} \partial \theta}+\frac{\beta}{R^{2}} \frac{\partial^{3}}{\partial \theta^{3}} \\
& L_{31}=\frac{v}{R} \frac{\partial}{\partial x} \\
& L_{32}=\frac{1}{R^{2}} \frac{\partial}{\partial \theta}-(2-v) \beta \frac{\partial^{3}}{\partial x^{2} \partial \theta}-\frac{\beta}{R^{2}} \frac{\partial^{3}}{\partial \theta^{3}} \\
& L_{33}=-\frac{1}{R^{2}}-2 \beta \frac{\partial^{4}}{\partial x^{2} \partial \theta^{2}}-R^{2} \beta \frac{\partial^{4}}{\partial x^{4}}-\frac{\beta}{R^{2}} \frac{\partial^{4}}{\partial \theta^{4}}-\frac{p_{h} \Phi}{R}-\frac{p_{h} \Phi}{R} \frac{\partial^{2}}{\partial \theta^{2}}-\rho h \Phi \frac{\partial^{2}}{\partial t^{2}}
\end{aligned}
$$


2.2 van der Waals interaction forces

To study the vibrational behavior of DWCNTs, a double-elastic shell model was developed that assumes each of the nested tubes in a CNT is an individual elastic shell, and the adjacent tubes are coupled to each other by normal vdW interactions. The pressures from vdW forces exerted on the inner and outer nanotubes through vdW interaction forces (Fig. 2 (b)) are given as

$p_{1}=c_{12}\left(\Delta w_{2}-\Delta w_{1}\right)$

$p_{2}=c_{21}\left(\Delta w_{1}-\Delta w_{2}\right)+p_{h}$

$\Delta w_{k}(k=1,2)$ are the radial displacements of the inner and outer nanotubes, and $c_{i j}(i, j=1$,

2) is the vdW interaction coefficient between nanotubes, which can be estimated from the Lennard-Jones potential [31]

$$
c_{i j}=\frac{\pi \varepsilon R_{j} \sigma^{6}}{3 a^{4}}\left[\frac{1120}{3} H_{i j}^{7}-1001 \sigma^{6} H_{i j}^{13}\right] \text {, }
$$

where

$$
H_{i j}^{m}=\left(R_{i}+R_{j}\right)^{-m} \int_{0}^{\frac{\pi}{2}} \frac{d \theta}{\left(1-K_{i j} \cos ^{2} \theta\right)^{\frac{m}{2}}} \quad(m=7,13)
$$

and

$$
K_{i j}=\frac{4 R_{i} R_{j}}{\left(R_{i}+R_{j}\right)^{2}}
$$

where $a$ is the carbon-carbon bond length $(0.142 \mathrm{~nm}), R_{i}$ and $R_{j}$ are the inner and outer radii of the DWCNTs, and $\sigma$ and $\varepsilon$ are the vdW radius and the well depth of the 
Lennard-Jones potential, respectively. The vdW parameters in the Lennard-Jones potential are $\varepsilon=2.967 \mathrm{meV}$ and $\sigma=0.34 \mathrm{~nm}$ as reported by Saito et al. [32].

Under pressure, the radial displacements of DWCNTs $\Delta w_{k}(k=1,2)$ in Eqs. $(14)-(15)$ are the function of the pressure exerted on the inner and outer tubes, given as

$\Delta w_{k}=\frac{p_{k} R_{k}^{2}}{E h}\left(1-\frac{v_{k}}{2}\right), \quad(k=1,2)$

where $v_{k}$ and $R_{k}(k=1,2)$ are Poisson's ratio and the radii of the inner and outer tubes, respectively.

Substituting Eq. (19) into Eqs. (14) - (15) yields

$p_{1}=\frac{p_{h}}{\Delta} \cdot \frac{c_{12} R_{2}^{2}}{E h}\left(1-\frac{v_{2}}{2}\right)$

$p_{2}=\frac{p_{h}}{\Delta} \cdot\left[1+\frac{c_{12} R_{1}^{2}}{E h}\left(1-\frac{v_{1}}{2}\right)\right]$

where

$\Delta=1+\frac{c_{12} R_{1}^{2}}{E h}\left(1-\frac{v_{1}}{2}\right)+\frac{c_{21} R_{2}^{2}}{E h}\left(1-\frac{v_{2}}{2}\right)$

\subsection{Frequency of the RBM of CNTs}

The general solution for the displacements $u, v$ and $w$ in the inner and outer tubes of a

DWCNT can be given by

$$
\begin{aligned}
& u=A_{k} \sin (n \theta) \cos \frac{m \pi x}{l} e^{i \omega t} \\
& v=B_{k} \cos (n \theta) \sin \frac{m \pi x}{l} e^{i \omega t}
\end{aligned}
$$


$w=C_{k} \sin (n \theta) \sin \frac{m \pi x}{l} e^{i \omega t} \quad(k=1,2)$

where $A_{k}, B_{k}$ and $C_{k}$ are the longitudinal, circumferential and radial amplitudes of displacement in the inner tube $(k=1)$ and the outer tube $(k=2)$, respectively. $\omega$ is the circular frequency of RBM and $t$ is time. The wave numbers $m$ and $n$ are the axial half-wave and circumferential numbers, respectively.

3. Numerical results and discussion

The influence of different nanotube parameters on the frequency of the RBM was investigated using the proposed method. An individual SWCNT is regarded to have the thickness of a graphene sheet, $0.34 \mathrm{~nm}$. In the calculations, the elastic modulus of a CNT is $3.3 \mathrm{TPa}$, Poisson's ratio is 0.27 , and the mass density of CNTs is $2.3 \mathrm{~g} / \mathrm{cm}^{3}$. DWCNTs were assumed to have an inner diameter of $2.2 \mathrm{~nm}$ and an outer diameter of $3.0 \mathrm{~nm}[3]$.

Using the present theoretical approach, the frequency of the RBM of isolated SWCNTs under no pressure as a function of radius was calculated and is shown in Fig.

3. The frequency of the RBM decreases significantly as the radius of the SWCNT increased from 0.3 to $1.0 \mathrm{~nm}$. Note that the commonly used unit for the frequency of the $\mathrm{RBM} f$ is in $\mathrm{cm}^{-1}$ for Raman spectroscopy experiments; the unit of Hertz (Hz) for $\omega$ has 
been adopted for convenience in this study. The relation between these terms is $\omega=\mathrm{c} \times f$, where $\mathrm{c}=3.0 \times 10^{8} \mathrm{~m} / \mathrm{s}$ and is the velocity of light in a vacuum. Some studies have used other approaches to research the frequency of the RBM of isolated SWCNTs. Kurti et al. [25] used first-principles calculations to show that the frequency of the RBM was 9.87 $-4.50 \mathrm{THz}$ for SWCNTs with radii of $0.35-0.78 \mathrm{~nm}$. Jorio et al. [33] calculated that the frequency of the RBM ranged from 5.28 to $4.32 \mathrm{THz}$ for SWCNTs with radii of 0.715 to $0.86 \mathrm{~nm}$ considering structural determination. Finite element methods [9] have also been used to investigate the RBM of CNTs and Dresselhaus et al. [34] published a review on the frequency of the RBM of isolated SWCNTs. The calculated frequencies of the RBM of SWCNTs with varying radius under no pressure agree closely with the values reported in the literatures, which verifies that the continuum elastic shell model accurately describes the frequency of the RBM of CNTs.

The frequencies of the RBM of the inner tube and outer tube of a DWCNT as a function of aspect ratio L/D are compared in Fig. 4. The changes in frequency for both inner and outer tubes show a sublinear logarithmic relationship with increasing aspect ratio. The frequencies of the RBM of the inner and outer tubes exhibit a concentrated distribution in the same region. Each tube exhibits the vibration frequency of CNTs over one terahertz, and the frequency of the inner tube is a little higher than that of the 
outer tube, except for a circumferential wave of $n=1$. The lowest frequency of DWCNTs is characterized by the circumferential wave $n=1$, and exists in the DWCNTs with larger aspect ratio. Meanwhile, the frequency of the RBM decreases dramatically with increasing aspect ratio. When the circumferential wave $n=1$, the cross sections of DWCNTs present away from the axial central axis, which makes the whole tubes to be unstable status. This phenomena is unique to DWCNTs with circumferential wave $n=1$, and is not observed in other circumferential waves. The unsteady DWCNTs with circumferential wave $n=1$ can be influenced by external factors more easily than with other circumferential waves. The frequency of the RBM of DWCNTs with an aspect ratio of $\mathrm{L} / \mathrm{D}<2$ decreases slightly with increasing aspect ratio. At aspect ratios of $\mathrm{L} / \mathrm{D}>2$, the frequency is almost independent of the aspect ratio when the circumferential wave $n$ is 2 or greater.

The frequencies of the RBM of DWCNTs with different circumferential wave numbers $(n=2,3)$, axial half-wave numbers $(m=1,3,5)$ and aspect ratios $(\mathrm{L} / \mathrm{D}=5,10$, 20) subjected to pressure were investigated and the results are shown in Figs. 5-7. The circumference wave number $n$ plays a critical role as the pressure increases, while the frequency of the RBM is hardly affected by the axial half-wave number or the aspect ratio. The frequency of the $\mathrm{RBM}$ has a positive linear relationship with increasing 
pressure in these figures, and this increase is more significant for the outer tube than the inner tube. Fig. 5 shows the frequencies of the RBM of inner and outer tubes with circumference numbers of $n=2$ and $n=3(m=4, \mathrm{~L} / \mathrm{D}=20)$ with increasing pressure. The gradients for frequency/pressure of the inner tube with $n=2$ and $n=3$ are 6.5 and 6.6 GHz/GPa, respectively, and 200.2 and $223.8 \mathrm{GHz} / \mathrm{GPa}$ for the outer tube, respectively. Frequencies of the RBM of the inner and outer tube with axial half-waves of $m=1, m=3$ and $m=5(n=2, \mathrm{~L} / \mathrm{D}=20)$ with increasing pressure are shown in Fig. 6. The gradients of the inner tube with $m=1, m=3$ and $m=5$ are $6.6,6.5$ and 6.4 $\mathrm{GHz} / \mathrm{GPa}$, respectively, and 204.9, 202.7 and 196.7 GHz/GPa for the outer tube, respectively. Fig. 7 shows the frequencies of the RBM of inner and outer tubes with aspect ratios of $\mathrm{L} / \mathrm{D}=5,10$ and $20(m=1, n=2)$ with increasing pressure. The gradients of the inner tube with $\mathrm{L} / \mathrm{D}=5,10$ and 20 are 6.5, 6.6 and $6.6 \mathrm{GHz} / \mathrm{GPa}$, respectively, and 200.3, 204.2 and $204.9 \mathrm{GHz} / \mathrm{GPa}$ for the outer tube, respectively. Therefore, according to the variation in the curves of the frequencies of the RBM frequencies of inner and outer tubes are almost parallel lines, and the range of variation in the RBM frequency of inner tube is smaller than that of outer tube. Considering unbundled DWCNTs, the trends and range of frequencies of the RBM for both the inner and outer tubes with increasing pressure agree closely with the experimental results of 
Arvanitidis and Christofilos [11], who revealed that the frequency of the RBM of the external tubes increased linearly with increasing pressure. Therefore, the theoretical results calculated in this study are consistent with experimental results. Moreover, due to vdW interaction forces and external pressure, the inner tube owns smaller variation of the RBM frequency than the outer tube. The inner tube is only reinforced by vdW interaction forces, and is slightly influenced by the external pressure, so the vdW interaction forces make a governing and positive role in the RBM frequency of the inner tube. Comparing with the inner tube, the outer tube suffers not only with vdW interaction forces, but also with external pressure which neutralizes the positive vdW interaction forces and makes a negative role at the same time. With the growing external pressure, the governing role of vdW interaction forces become less and less, which leads that the RBM frequency of the outer tube is more sensitive to the external pressure and has a bigger variation of the RBM frequency than that of the inner tube.

\section{Conclusions}

The RBM of DWCNTs subjected to pressures were investigated using an elastic continuum mechanics model. The analysis was based on a continuum mechanics model where each tube of a DWCNT was described as an individual elastic shell and 
DWCNTs were considered to be two-layered nanotube shells coupled by vdW interactions. The axial half-wave number and the aspect ratio had little effect on the frequency of the RBM. In contrast, the circumference wave number affected the frequency of the RBM significantly. Compared with previous experimental and simulation investigations on the frequency of the RBM of isolated SWCNTs with increasing radius and DWCNTs with increasing pressure, the continuum shell model can be used to reasonably predict the frequency of the RBM of CNTs subjected to pressure. According to the variation of the frequency of the RBM of DWCNTs with increasing pressure, the inner tube is affected less than the outer tube. Based on this model and a theoretical approach, a numerical simulation investigating the RBM of multiwalled CNTs subjected to pressure can be developed. Theoretical analysis of the RBM of DWCNTs is useful for nanofabrication and nanodevice technologies.

\section{Acknowledgments}

This work was supported by a Grant-in-Aid from the Global COE Program from the

Ministry of Education, Culture, Sports, Science and Technology and by CLUSTER (second stage) from the Ministry of Education, Culture, Sports, Science and Technology (Japan). 


\section{Figure captions}

Figure 1 Schematic diagram showing the cylindrical coordinates of the CNT model used for analysis

Figure 2 DWCNT model subjected to pressure for analysis

Figure 3 Frequency of the RBM of SWCNTs not subjected to pressure as a function of radius

Figure 4 Comparison of the frequencies of the RBM of inner and outer tubes in DWCNTs subjected pressure with different circumference wave numbers as a function of aspect ratio $(m=1)$

Figure 5 Comparison of the frequencies of the RBM between inner and outer tubes in DWCNTs with different circumference wave numbers as a function of pressure $(m=4, \mathrm{~L} / \mathrm{D}=20)$

Figure 6 Comparison of the frequencies of the RBM between inner and outer tubes in DWCNTs with different axial half-wave numbers as a function of pressure ( $n$ $=2, \mathrm{~L} / \mathrm{D}=20)$

Figure 7 Comparison of the frequencies of the RBM between inner and outer tubes in DWCNTs with different aspect ratios as a function of pressure $(m=1, n=2)$ 


\section{References}

[1] J. Yoon, C.Q. Ru, A. Mioduchowski, Comput. Sci. Technol. 63 (2003) 1533-42.

[2] Y. Zhang, G. Liu, X. Han, Phys. Lett. A 340 (2005) 258-266.

[3] T. Natsuki, X.W. Lei, Q.Q Ni, M. Endo, Phys. Lett. A 374 (2010) 2670-74.

[4] L.L. Ke, Y. Xiang, J. Yang, S. Kitipornchai, Comput. Mater. Sci. 47 (2009) 409-417.

[5] C.M. Wang, V.B.C. Tan, Y.Y. Zhang, J. Sound Vib. 294 (2006) 1060-72.

[6] T. Natsuki, X.W. Lei, Q.Q Ni ,M. Endo, Phys. Lett. A 374 (2010) 4684-89.

[7] M.S. Dresselhausa, G. Dresselhausb, R. Saitoc, A. Joriod, Physics Reports 409 (2005) 47-99.

[8] S. Costa, E. Borowiak-Palen, M. Kruszyñska, A. Bachmatiuk, R J. Kaleńczuk, Mater. Sci. Poland 26 (2008) 433-441.

[9] H.C. Cheng, Y.L. Liu, C.H. Wu, W.H. Chen, Comput. Methods Appl. M. 199 (2010) 2820-7.

[10] I. Loa, J. Raman Spectrosc. 34 (2003) 611-627.

[11] J. Arvanitidis, D. Christofilos, Phys. Stat. Sol. (b) 244 (2007) 127-135.

[12] U. Schlecht, U.D. Venkateswaran, E. Richter, J. Chen, R.C. Haddon, P. C. Eklund, and A. M. Rao, J. Nanosci. Nanotech. 3 (2003) 139-143.

[13] U.D. Venkateswaran, A.M. Rao, E. Richter M. Menon, A. Rinzler and R.E. Smalley, 
P.C. Eklund, Phys. Rev. B 59 (1999) 10928-34.

[14] U.D. Venkateswaran, E.A. Brandsen, U. Schlecht, A.M. Rao, E. Richter, I. Lo, K. Syassen and P.C. Eklund, Phys. Stat. Sol. (b) 223 (2001) 225-235.

[15] S. Lebedkin, K. Arnold, O. Kiowski, F Hennrich and M.M. Kappes, Phys. Rev. B 73 (2006) 094109-1-12.

[16] A.M. Rao, E. Richter, S. Bandow, B. Chase, P.C. Eklund, K.A. Williams, S. Fang, K.R. Subbaswamy, M. Menon, A. Thess, R.E. Smalley, G. Dresselhaus and M. S. Dresselhaus, Science 275 (1997) 187-191.

[17] S. Bandow, S. Asaka, Y. Saito, A.M. Rao, L. Grigorian, E. Richter and P.C. Eklund, Phys. Rev. Lett. 80 (1998)3779-82.

[18] A.K. Sood, P.V. Teresdesai, D.V.S. Muthu, R. Sen, A. Govindaraj and C.N.R. Rao, Phys. Status Solidi B 215 (1999) 393-401.

[19] M.J. Peters, L.E. McNeil, J.P. Lu, and D. Kahn, Phys. Rev. B 61 (2000) 5939-44.

[20] A.M. Rao, P.C. Eklund, S. Bandow, A. Thess and R.E. Smalley, Nature 388 (1997) 257-259.

[21] A.M. Rao, J. Chen, E. Richter, U. Schlecht, P.C. Eklund, R.C. Haddon, U.D. Venkateswaran, Y.K. Kwon and D. Tománek, Phys. Rev. Lett. 86 (2001) 3895-98.

[22] V. Gadagkar, S. Saha, D.V.S. Muthu, P.K. Maiti, Y.Lansac, A. Jagota, A. Moravsky, 
R.O. Loutfy and A.K. Sood, J. Nanosci. Nanotechnol. 7 (2007)1753-71.

[23] P. Puech, H. Hubel, D.J. Dunstan, R.R. Bacsa, C. Laurent and W.S. Bacsa1, Phys. Rev. Lett. 93 (2004) 095506-1-4.

[24] P. Puech, H. Hubel, D.J. Dunstan, A. Bassil, R. Bacsa, A. Peigney, E. Flahaut, C. Laurent and W.S. Bacsa, Phys. Stat. Sol. (b) 241(2004) 3360-66.

[25] J. Kurti, G. Kresse, H. Kuzmany, Phys. Rev. B 58 (1998) 8869-72.

[26] H.M. Lawler, D. Areshkin, J.W. Mintmire and C.T. White, Phys. Rev. B 72 (2005) 233403-1-4.

[27] C.Y. Wang, C.Q. Ru and A. Mioduchowski, J. Appl. Phys. 97 (2005) 024310-1-10.

[28] T. Natsuki, T. Hayashi, M. Endo, Appl. Phys. A 83 (2006) 13-17.

[29] T. Natsuki, N. Fujita, Q.Q. Ni, M. Endo, J. Appl. Phys. 106 (2009)084310-1-5.

[30] S.P. Timoshenko, J.M.Gere, Theory of elastic stability, second ed., McGraw-Hill, New York, 1961.

[31] X.Q. He, S.Kitipornchai, K.M. Liew, J. Mech. Phys. Solids 53 (2005) 303-326.

[32] R. Saito, R. Matsuo, T. Kimura, G. Dresselhaus, M.S. Dresselhaus, Chem. Phys. Lett. 348, (2001) 187-193

[33] A. Jorio, R. Saito, J.H. Hafner, C.M. Lieber, M. Hunter, T. McClure, G. Dresselhaus and M.S. Dresselhaus, Phys. Rev. Lett. 86 (2001) 1118-21. 
[34] M.S. Dresselhaus, G. Dresselhaus, A. Joriob, A.G. Souza Filhob, R. Saitof, Carbon 40 (2002) 2043-61. 
Fig.1

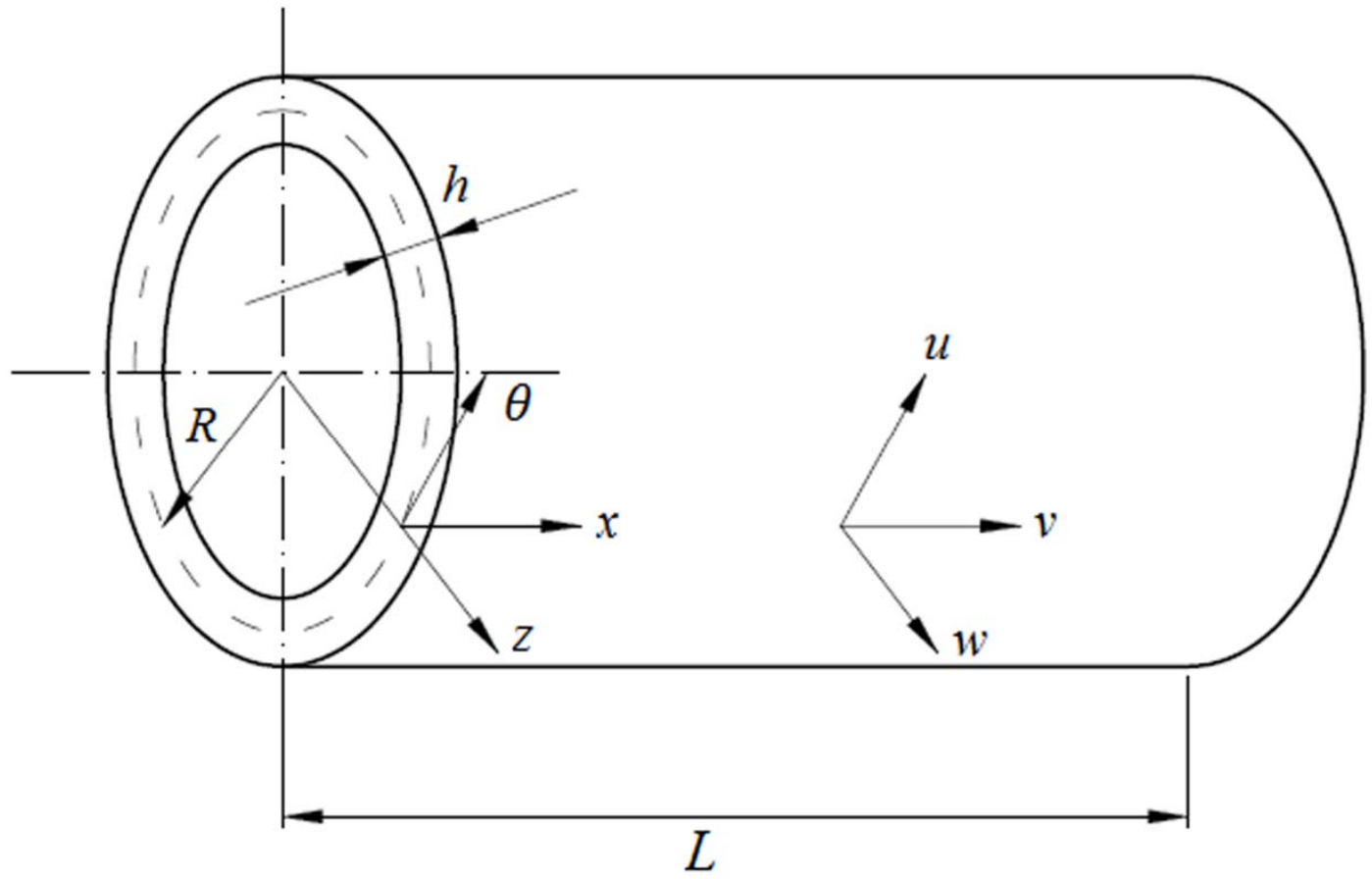

Fig.2
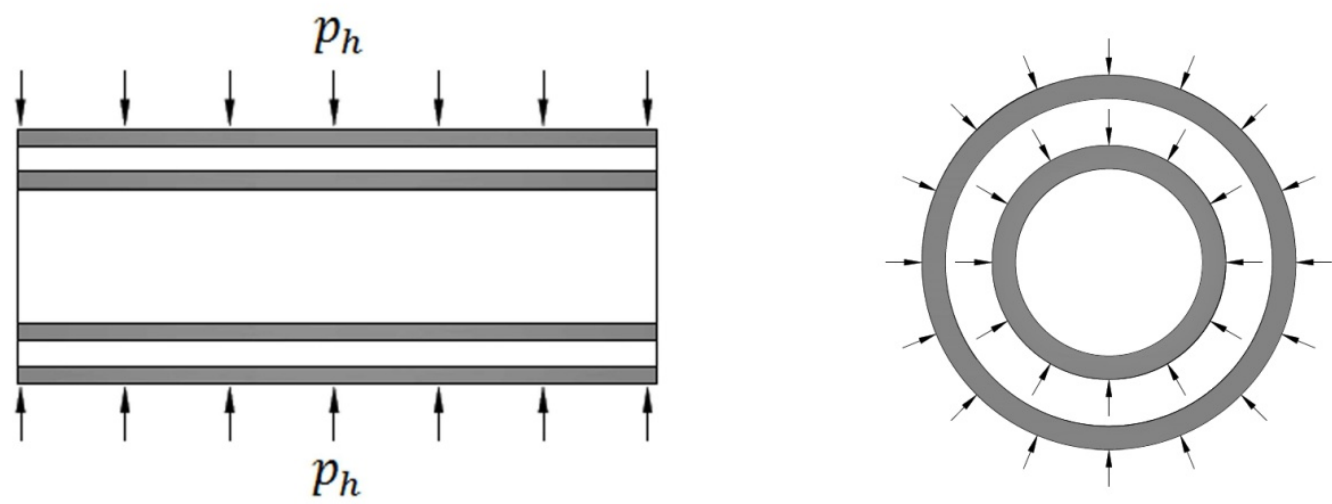

(a)

(b) 
Fig. 3

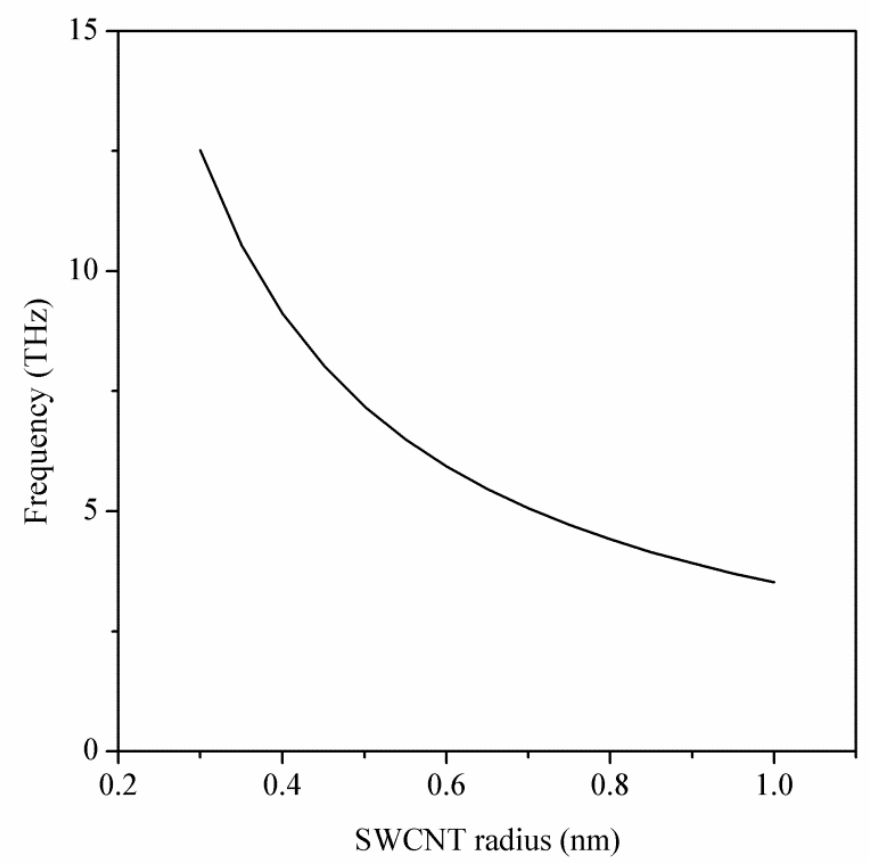

Fig.4

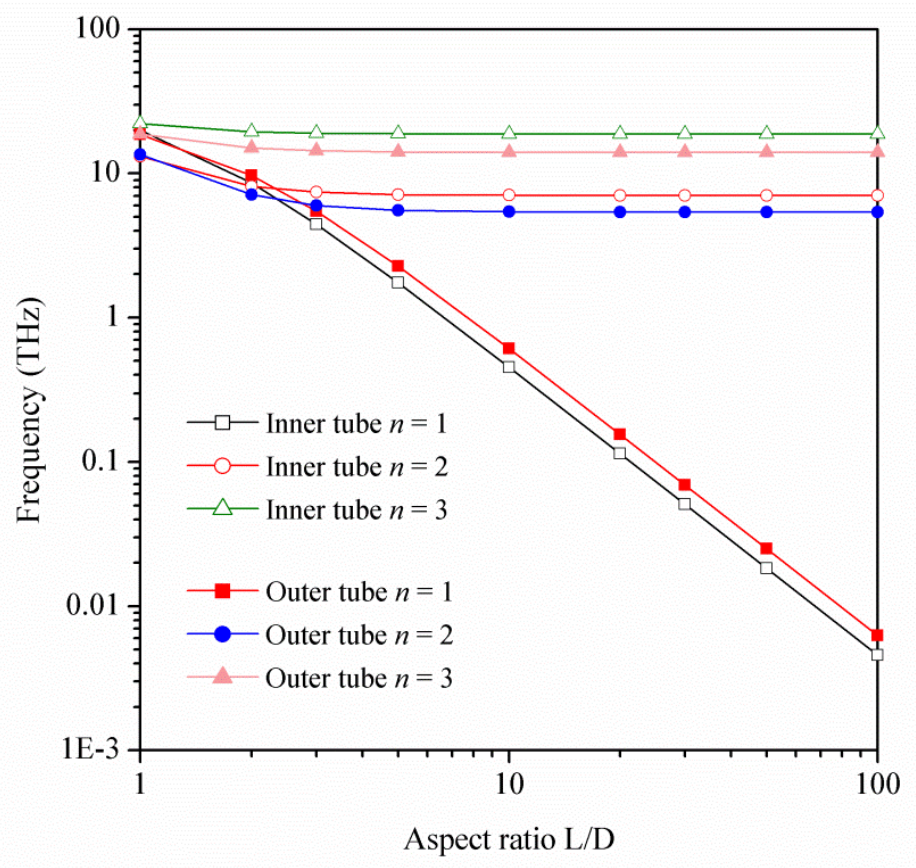


Fig.5

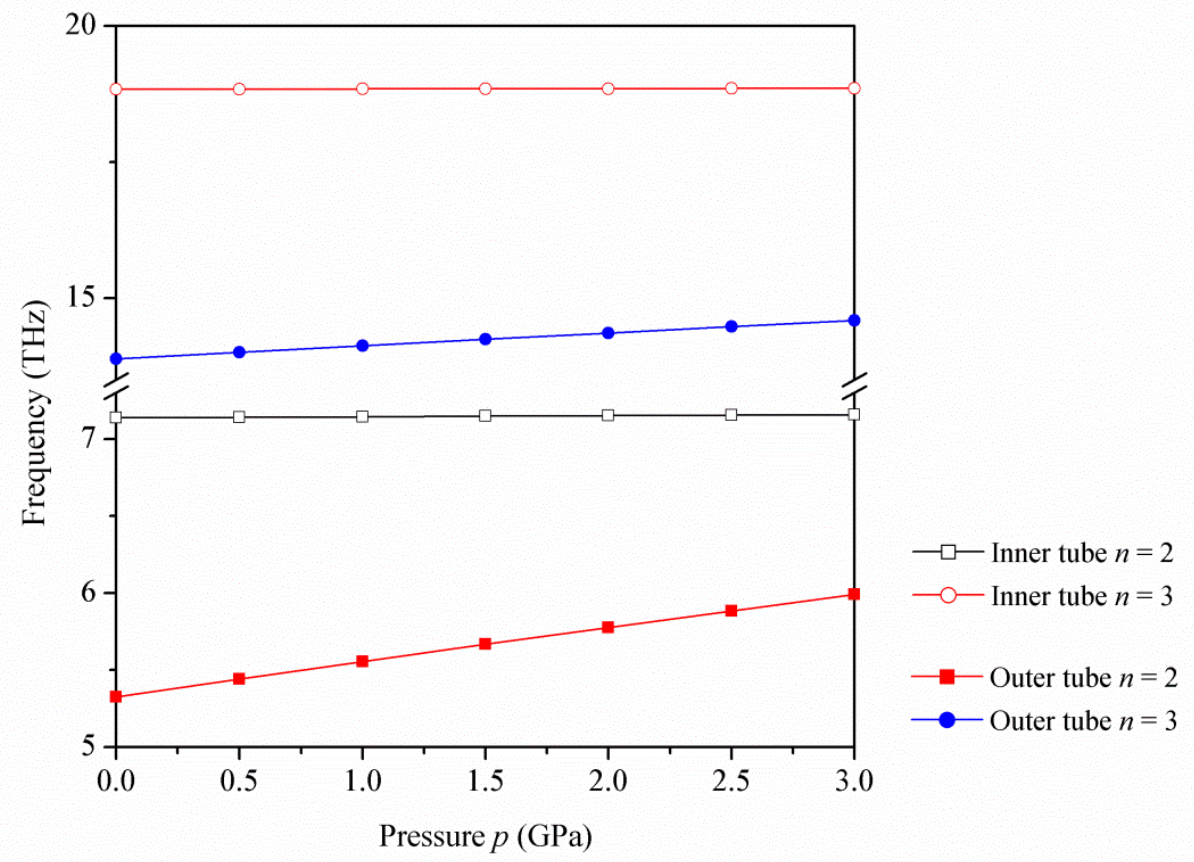

Fig.6

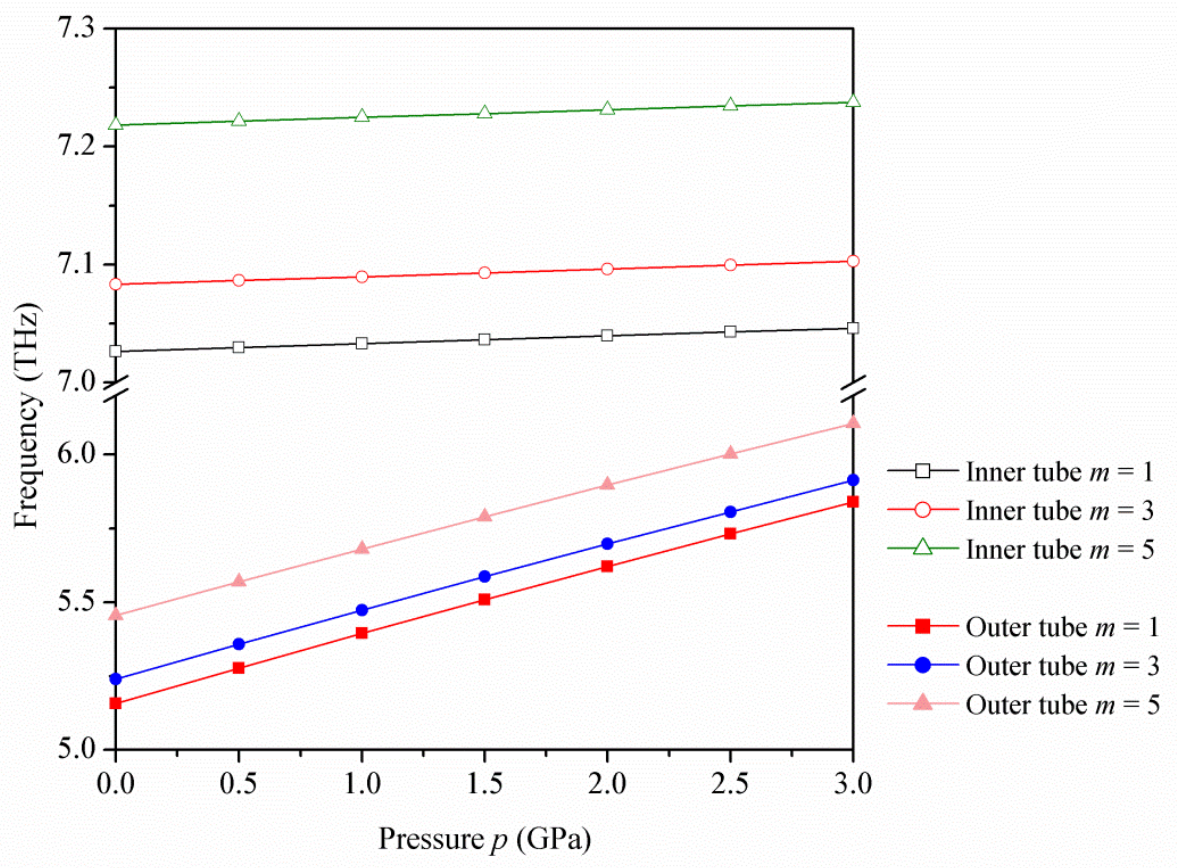


Fig.7

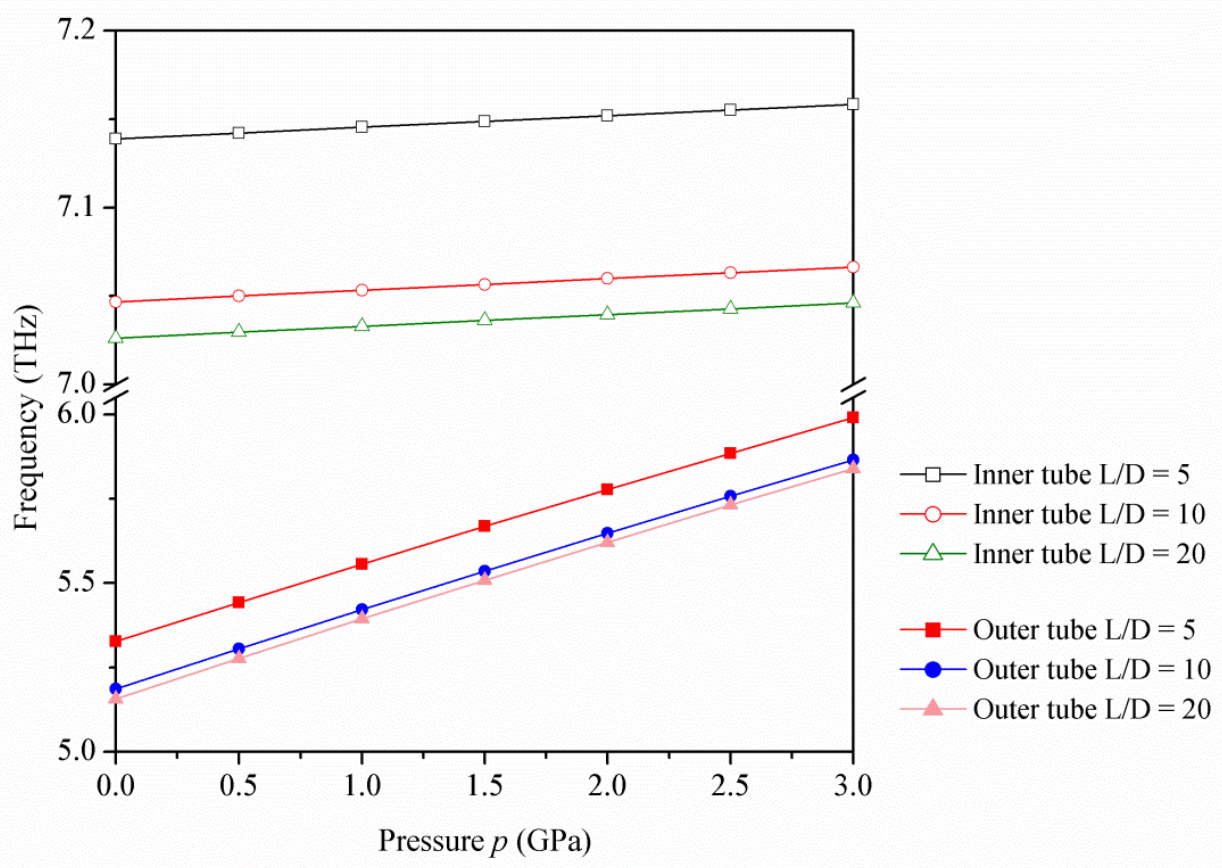

